\title{
La familia y el juego como estrategia de aprendizaje a distancia durante la pandemia del Covid-19 en México: Una propuesta desde la enseñanza universitaria en ciencias de la salud
}

\author{
The family and the play as a distance learning strategy during the \\ pandemic of Covid-19 in México: A proposal from university \\ education in health sciences
}

\author{
Giovanna Georgina Ramírez Cerón \\ Universidad de Guadalajara. Guadalajara, México \\ giovanna.ramirez@academicos.udg.mx
}

\begin{abstract}
Resumen
La pandemia por Covid-19 ha impactado las formas de aprender, por lo que el cambio repentino de la modalidad presencial a la educación a distancia como medida para prevenir contagios, es un fenómeno que debe ser investigado para brindar alternativas que apoyen el proceso de aprendizaje desde casa. La presente investigación tiene por objetivo diseñar, aplicar, evaluar y proponer estrategias de aprendizaje para que los estudiantes alcancen su máximo potencial por medio de la educación a distancia durante la pandemia. Para ello se llevó a cabo una investigación-acción educativa con ciento veinte estudiantes de ciencias de salud (enfermería y terapia física), de la Universidad de Guadalajara, durante los meses de marzo a octubre de 2020. Las estrategias de aprendizaje se aplicaron en la modalidad a distancia debido a la suspensión de clases presenciales por el Covid19 y se basaron en la participación de la familia y la introducción del juego en las actividades de aprendizaje del curso de etimologías médicas. La evaluación de las estrategias se llevó a cabo mediante la recogida de las evidencias de los aprendizajes y un cuestionario virtual de autoevaluación de las mismas. Se encontró que incluir a la familia y el juego en la educación a distancia ayudó a mitigar los efectos negativos del confinamiento por la pandemia, lo cual, además ha fortalecido la resiliencia de los estudiantes.

Palabras clave: Estudiantes universitarios, aprendizaje a distancia, aprendizaje lúdico, familia, pandemia, resiliencia.
\end{abstract}

\begin{abstract}
The pandemic by Covid-19 has impacted the ways of learning, so that the sudden change from the face to face modality to distance education as a measure to precent contagion, it is a phenomenon that must be investigated to provide alternatives that support the learning process from home. The aims this present study are design, apply, evaluate and propose learnig strategies for students to reach their máximum potential through distance education during the pandemic. For it an educational action research was carried out with one hundred and twenty health science students (nursing and physioterapy) from the Universidad de Guadalajara, during March to October 2020. Distance learning strategies were carried because out to the suspension of face to face clases due to Covid-19, wich they were based on family participation and the insertion on the play in the learning activities of the
\end{abstract}


medical etymologies course. The evaluation of the strategies was carried out by collecting the evidence of learning and a virtual self-evaluation questionnaire. It was found that including family and play in distance education helped mitigate the negative effects of confinement from the pandemic, wich has also strengthened the resilience of students.

Keywords: University students, distance learning, playful learning, family, pandemic, resilience.

\section{Introducción}

Hacia finales de febrero de 2020 la Secretaría de Salud de México dio a conocer que se había dado el primer contagio por Covid-19 en el país. Días después de este hallazgo, debido al comportamiento que estaba presentando la pandemia, el Gobierno Federal determinó que a partir del 23 de marzo de 2020 serían suspendidas las clases presenciales en todos los niveles educativos como una medida para disminuir los contagios, además de una interrupción de actividades no esenciales y de eventos de congregación masiva (Secretaría de Gobernación, 2020a). En el Estado de Jalisco, México, a partir de un análisis sobre la situación sanitaria, el Gobernador Enrique Alfaro Ramírez determinó que la suspensión de clases presenciales en el nivel de educación básica se llevaría a cabo una semana antes en comparación con el resto del país, es decir, a partir del martes 17 de marzo de 2020 (Gobierno del Estado de Jalisco, 2020). Por su parte, la Universidad de Guadalajara, la máxima casa de estudios de este Estado, emitió por medio de un comunicado que a partir del 17 de marzo de 2020 serían suspendidas las clases presenciales tanto en el nivel medio superior, como en el superior y que para dar continuidad a la formación académica durante la contingencia sanitaria, sería puesta a disposición de la comunidad universitaria la plataforma Google G Suite for Education, así como la difusión de vídeos tutoriales para hacer el mejor uso de dicha herramienta didáctica (Universidad de Guadalajara, 16 de marzo de 2020).

En Jalisco, tanto para las instituciones educativas, como para el profesorado y el alumnado, el reto de adaptarse a una modalidad de educación a distancia durante los primeros meses de la pandemia por Covid-19 fue mayor que en otros Estados de la Republica Mexicana, ya que la suspensión de clases en la modalidad presencial fue dada de forma repentina, dando muy poco tiempo para el desarrollo de una planeación y diseño de estrategias de aprendizaje para esta primera etapa de la pandemia. La información de dicha suspensión, se proporcionó en el contexto de un fin de semana largo o puente del lunes 16 de marzo de 2020, día de asueto para el país, debido a que se conmemora el natalicio de Benito Juárez. Por lo que el viernes 13 de marzo del mismo año, profesores y estudiantes de todos los niveles educativos se presentaron a sus clases presenciales con "toda normalidad" y fue en el transcurso de ese viernes que se informó a la ciudadanía que el martes 17 de marzo ya no se presentarían niñas, niños, adolescentes y jóvenes a sus escuelas. Muchos se despidieron aquel viernes como lo hacían en cualquier otro día, esperando volverse a ver "presencialmente" al regreso del puente. Pero para los jaliscienses, por su propia seguridad y las de sus familias esto no sería posible y hasta el día de hoy no sé tiene certeza sobre cuando será el regreso presencial a las aulas. Lo anterior, se debe a que en México, las autoridades en materia

La familia y el juego como estrategia de aprendizaje a distancia durante la pandemia del Covid19 en México: Una propuesta desde la enseñanza universitaria en ciencias de la salud. Giovanna Georgina Ramírez Cerón. 
de salud determinaron una serie de medidas preventivas para la contención de contagios por Covid-19, una de estas fue la Jornada Nacional de Sana Distancia, misma que se extendió del 23 de marzo de 2020 (Secretaría de Gobernación, 2020a), hasta el 30 de mayo del mismo año (Secretaría de Gobernación, 2020b). Al terminar esta Jornada, dio inicio el proceso de regreso a la "nueva normalidad", para lo cual la Secretaría de Salud (2020), diseñó el semáforo de riesgo epidemiológico, el cual es un medio para regular el uso del espacio público conforme al riesgo de contagio por Covid-19. Este es conformado por los colores ${ }^{1}$ rojo, naranja, amarillo y verde, y se determina de acuerdo a la situación particular de cada Estado del país. La actividad educativa presencial en México, solo se llevará a cabo cuando el semáforo epidemiológico se encuentre en color verde y hasta el mes de octubre de 2020, Jalisco solo ha podido alcanzar el color naranja; pese a que en dicho Estado se han tomado medidas enérgicas para mantener a la ciudadanía en casa como el cierre de bares, casinos, antros, cantinas, centros nocturnos y salones de fiesta (Periódico Oficial del Estado de Jalisco, 17 de marzo de 2020), además del uso obligatorio del cubrebocas o mascarilla en la calle y en múltiples establecimientos; observándose arrestos por no usar la mascarilla (Blanco, 26 de abril de 2020).

El cierre masivo de las actividades educativas presenciales como una medida para disminuir los contagios por Covid-19 ha afectado a más de 190 países; en la región de América Latina y el Caribe se ha optado por implementar modalidades de aprendizaje a distancia para responder al desafío de dar continuidad a las trayectorias educativas, el uso de plataformas virtuales de aprendizaje sincrónico y asincrónico, así como el de programas educativos a través de la radio y la televisión han sido el medio para que alumnos y profesores puedan encontrarse (CEPAL-UNESCO, 2020). En este panorama, el papel de la Internet ha sido importante ya que ha a través de la misma se ha podido contar con una cantidad importante de recursos pedagógicos, así como de herramientas comunicacionales que acercan la escuela a los hogares de los estudiantes que se encuentran en confinamiento ocasionado por la pandemia, pero también se han visibilizado las desigualdades que se dan en el acceso a estas plataformas (CEPALUNESCO, 2020).

\footnotetext{
${ }^{1}$ Los colores del semáforo epidemiológico (Secretaría de Salud, 2020) así como las actividades permitidas de acuerdo al mismo son:
}

Rojo: solo se permiten actividades económicas esenciales.

Naranja: se permiten actividades económicas esenciales y las empresas con actividades económicas no esenciales podrán trabajar con el 30\% del personal, se abrirán espacios públicos con una cantidad reducida de personas.

Amarillo: todas las actividades laborales son permitidas, el espacio público abierto se abre de forma regular y los espacios públicos cerrados se abren con una reducida cantidad de personas.

Verde: todas las actividades están permitidas, incluidas las escolares.

Durante todos los colores se deben seguir las medidas de higiene y el cuidado especial de la población vulnerable.

La familia y el juego como estrategia de aprendizaje a distancia durante la pandemia del Covid19 en México: Una propuesta desde la enseñanza universitaria en ciencias de la salud. Giovanna Georgina Ramírez Cerón. 
El uso de las tecnologías de la información y la comunicación, ya formaba parte de diversos procesos de enseñanza-aprendizaje mucho antes de la pandemia por Covid-19. Crovi (2006) afirmaba que las tecnologías han transformado las formas tradicionales de enseñanza, Mendiguren, Meso y Pérez (2012) coinciden en que a través de las computadoras e Internet se hace posible la educación a distancia, donde las redes sociales virtuales han cobrado una especial relevancia en el ámbito educativo. Un ejemplo de esto, es «Proyecto Facebook», coordinado por Piscitelli y Adaime (2010), en Buenos Aires Argentina, el cual surgió hace poco más de una década, con el objetivo de crear un ambiente colaborativo y abierto en la educación, para que los alumnos fueran coparticipes de su proceso educativo.

El papel de las tecnologías de la información y la comunicación en el ámbito educativo no es el problema central al que se enfrentan profesores y estudiantes durante la pandemia por Covid-19, el principal desafío es aprender en confinamiento, saliendo de casa solo para lo estrictamente necesario, en medio de un ambiente en el que la economía a nivel mundial se ha visto devastada, donde en las familias de muchos de los estudiantes se vive la pérdida de empleos, la falta de acceso a la tecnología necesaria para continuar con las clases a distancia, enfermedad e incluso contagios por Covid-19 o hasta la muerte por el mismo, además de las repercusiones psicosociales que trae consigo la ausencia de contacto físico y el aislamiento; como afirman Ramírez-Ortiz, Castro-Quintero, Lerma-Córdoba, Yela-Ceballos y Escobar-Córdoba (2020, p. 8) "En la situación actual de confinamiento global en el hogar debido al brote del COVID-19, la mayoría de las personas están expuestas a situaciones estresantes sin precedentes y de duración desconocida".

El impacto psicológico, social y educativo que se ha tenido como consecuencia del cierre de las escuelas como medida de prevención de contagios por Covid-19, así como la incertidumbre que se sigue experimentando en torno al rumbo que vaya a seguir esta pandemia y de las formas de enseñanza-aprendizaje que se deban adoptar en las siguientes fases de la misma, exige la generación de investigaciones científicas que ayuden a la comprensión del fenómeno, así como del desarrollo de propuestas que hagan frente a la pandemia en el ámbito educativo en el que se están reconfigurado las formas de aprender. Partiendo de lo anteriormente planteado surge la necesidad de realizar la presente investigación, la cual tiene como objetivos:

1. Diseñar, aplicar y evaluar estrategias de aprendizaje en las que se incluya el juego y la participación de la familia de estudiantes universitarios durante el confinamiento como prevención de contagios de Covid-19; para que estos alcancen su máximo potencial en la competencia a desarrollar en la asignatura de etimologías médicas a pesar de aprender desde casa durante la pandemia.

2. Aportar información y metodologías que ayuden a la comprensión de los procesos educativos durante la pandemia por Covid-19 desde la investigación-acción educativa.

3. Proponer estrategias de aprendizaje que puedan ser aplicadas en la educación a distancia debido a la pandemia por Covid-19, así como en las etapas posteriores de la misma.

La familia y el juego como estrategia de aprendizaje a distancia durante la pandemia del Covid19 en México: Una propuesta desde la enseñanza universitaria en ciencias de la salud. Giovanna Georgina Ramírez Cerón. 
Antes de presentar la estrategia metodológica, es importante mencionar que cuando se diseña una estrategia de aprendizaje es necesario que ésta se enmarque dentro de un modelo de aprendizaje, entre los principales modelos se encuentran el cognitivo, el conductual y el constructivista, y desde este último se lleva a cabo la propuesta educativa que se pretende aportar con la presente investigación.

El modelo constructivista puede ser considerado uno de los más influyentes, desde su aportación teórica hasta su práctica pedagógica (Olmedo y Farrenons, 2017). El cual concibe al alumno como el responsable de su propio aprendizaje, y entiende este último como un proceso de construcción, más que de transmisión de conocimientos, por tanto el docente desempeña el rol de facilitador de las condiciones necesarias para lograr que los alumnos lleven a cabo sus actividades constructivistas (Olmedo y Farrenons, 2017). La educación a distancia y más en condiciones de pandemia, requiere de una visión constructivista del aprendizaje. Ya que como refiere Ponce (2006), esta modalidad se centra en los procesos del aprendizaje, por lo que requiere que las y los estudiantes sean autónomos en sus procesos de construcción del conocimiento, lo cual implica una actitud participativa, proactiva, crítica, reflexiva, organizada y colaborativa por parte de los mismos (Ponce, 2016). El aprendizaje autónomo, es aquel en el que la o el alumno es consciente de su proceso educativo y conoce sus propios estilos y formas en que aprende mejor, por tanto, desde esta perspectiva el profesor se convierte en facilitador y motivador para que el alumnado alcance el mayor aprendizaje (Montes de Oca, 2007).

Vygotski es un destacado precursor del modelo constructivista y desde este autor es posible comprender que el estudiante universitario no puede limitar su aprendizaje al espacio físico de la escuela, ya que fuera de ésta, ha tenido diversos acercamientos a la realidad que le dan soporte a los aprendizajes que elabora en el aula. La interacción con el "otro" es necesaria para que el alumno construya sus aprendizajes y así alcance un mayor potencial. En este sentido Vigotsky (1979; 2009: p.133), desarrolla el concepto de Zona de Desarrollo Próximo y la define como "la distancia entre el nivel real de desarrollo, determinado por la capacidad de resolver independientemente un problema, y el nivel de desarrollo potencial, determinado a través de la resolución de un problema bajo la guía de un adulto o en colaboración con otro compañero más capaz”. Cuando el estudiante aprende en casa, su familia y las personas con quienes convive, se convierten en sus compañeros de clase e incluso en sus profesores, partiendo del rol que estos desempeñan en la construcción del aprendizaje. Además del papel en la educación que puede tener la familia del estudiante, la interacción con esta puede mitigar los efectos psicosociales del confinamiento.

El juego tiene un valor educativo en todos los grados escolares, ya que motiva el estudio, facilita la adquisición de conocimientos, fomenta la cohesión y la solidaridad entre los participantes, además de que propicia la tolerancia, el respeto y la responsabilidad (Bernabeu y Goldstein, 2009), y favorece la construcción de relaciones interpersonales y de amistad en el área educativa (Ortíz, 2009), lo cual en momentos de confinamiento, incertidumbre, problemas económicos, familiares, emocionales y sanitarios, puede llevar a que el estudiante tenga un mayor aprovechamiento escolar. Para Vygotski (1979; 2009), el juego es parte importante del aprendizaje, además de que satisface ciertas necesidades, impulsa la imaginación, la asimilación de reglas y construye afectividades, a pesar de en ocasiones genere frustración, pero también placer.

La familia y el juego como estrategia de aprendizaje a distancia durante la pandemia del Covid19 en México: Una propuesta desde la enseñanza universitaria en ciencias de la salud. Giovanna Georgina Ramírez Cerón. 


\section{Estrategia metodológica}

\section{El diseño metodológico}

Se realizó el presente trabajo desde una lógica cualitativa de investigación, con un diseño de investigación-acción educativa. De acuerdo con Elliott (2005), esta modalidad de investigación la realizan las y los docentes a partir de problemáticas que identifican en su práctica con el objeto de comprender lo que sucede y así encontrar una acción adecuada de intervención. La forma sugerida para llevar a cabo la investigación a través de esta propuesta es realizar descripciones de situaciones concretas en un ambiente natural, por lo que los estudios de casos pueden ser útiles en este sentido.

\section{Los participantes}

La Universidad de Guadalajara se conforma por la Red Universitaria de Jalisco y está integrada por centros universitarios temáticos los cuáles se ubican en la zona metropolitana de Guadalajara, los centros universitarios regionales, el sistema de educación media superior y el sistema de universidad virtual. El Centro Universitario de Ciencias de la Salud (CUCS) forma parte de los centros universitarios temáticos de esta casa de estudios. Este tiene como misión mejorar las condiciones de salud por medio de la formación de recursos humanos con un alto nivel de competencia profesional, capaz de aplicar el conocimiento científico en pro de la ciudadanía, vinculándose con la sociedad para alcanzar el bien de la comunidad, por lo que en este espacio universitario se ofertan las licenciaturas de medicina, enfermería, nutrición, psicología, cirujano dentista, las carreras técnico superior universitario en prótesis dental, terapia respiratoria, terapia física y radiología e imagen, así como la carrera técnica en enfermería, entre otras (Centro Universitario de Ciencias de la Salud, 2020). La formación de profesionales de la salud requiere el acercamiento a la realidad a través de la interacción directa con la población, así como la puesta en práctica de los saberes teóricos y formativos. El aprendizaje a distancia en medio de la pandemia por Covid-19 no ha sido una tarea fácil para nadie, pero en lo referente a la formación de los profesionales de la salud, el reto de aprender desde casa durante esta pandemia es aún mayor, pues en las manos de estas y estos universitarios estará la vida de muchas personas, así como su recuperación y mejora de la calidad de vida.

Participaron en la investigación cuatro grupos de estudiantes de la asignatura de etimologías médicas del Centro Universitario de Ciencias de la Salud de la Universidad de Guadalajara. Tres de ellos cursaron la materia en el ciclo escolar 2020-A y uno en el ciclo 2020-B. En ambos calendarios se impartieron las clases a distancia desde el primer hasta el último día del curso debido a las medidas de prevención de contagios por Covid-19. Tres de los grupos pertenecen a la carrea de enfermería y uno a la carrera de técnico superior universitario en terapia física.

El número total de los participantes del estudio fue de 120, de los cuales 89 pertenecen a la carrera de enfermería y 31 a la de técnico superior universitario en terapia física; 45 son hombres y 75 mujeres, sus edades oscilan entre los 18 y 28 años de edad, y 5 de estos son padres y madres de familia (Ver tabla número 1). La elección de los mismos fue por conveniencia, considerando a los grupos de los cuales es profesora de

La familia y el juego como estrategia de aprendizaje a distancia durante la pandemia del Covid19 en México: Una propuesta desde la enseñanza universitaria en ciencias de la salud. Giovanna Georgina Ramírez Cerón. Página $6 \mid 20$ 
etimologías médicas la autora de la presente investigación y que tomaron el curso a distancia por la pandemia por Covid-19.

\section{Tabla 1}

Características de los participantes del estudio

\begin{tabular}{llll}
\hline & Enfermería & Terapia física & Total \\
\hline Hombres & 31 & 14 & 45 \\
\hline Mujeres & 58 & 17 & 75 \\
\hline Total & 89 & 31 & 120 \\
\hline
\end{tabular}

Nota: Elaboración propia

\section{Procedimiento}

1.- Diseño de la propuesta: En el Centro Universitario de Ciencias de la Salud de la Universidad de Guadalajara, se ofrecen algunos cursos que son llamados por profesores y alumnos como "espejo". Este nombre obedece a que su duración solo abarca la mitad del ciclo escolar, por lo que se ofertan dos materias en el mismo horario durante el semestre, de tal forma que al terminar un curso comienza a la siguiente semana su "espejo". El ciclo escolar 2020-A, abarcó los meses de enero a mayo de 2020, durante este ciclo dio comienzo la suspensión de clases presenciales como medida preventiva y los cursos "espejo" en el Centro Universitario de Ciencias de la Salud dieron inicio el 17 de marzo de 2020, justo el día en que fueron suspendidas las clases en su modalidad presencial. Por tanto en las primeras horas que se dio este anuncio profesores y estudiantes tenían la incertidumbre de saber cómo trabajarían y cómo se contactarían, ya que se supone se conocerían el día en que ya no pudieron presentarse a la universidad. Sin embargo, en cuestión de horas, el equipo de la Universidad de Guadalajara, proporcionó los datos de contacto tanto de profesores y estudiantes para poder establecer la comunicación e iniciar los cursos en la modalidad a distancia.

En esa situación la autora de la presente investigación, se dio a la tarea de diseñar las estrategias que se llevarían a cabo en el curso de etimologías médicas, haciendo las adaptaciones necesarias de la modalidad presencial a la virtual. Considerando el contexto planteado en la introducción de este trabajo, por lo que se consideró la participación de la familia, amigos o pareja en las actividades de aprendizaje, para mitigar los efectos del aislamiento y se recurrió al aprendizaje autónomo, aprendizaje lúdico y el aprendizaje por casos, mismos que ya eran implementados por la autora en la modalidad presencial previa a la pandemia por Covid-19.

La familia y el juego como estrategia de aprendizaje a distancia durante la pandemia del Covid19 en México: Una propuesta desde la enseñanza universitaria en ciencias de la salud. Giovanna Georgina Ramírez Cerón. 
2.- Desarrollo de la propuesta: Una vez diseñadas las estrategias de aprendizaje se procedió a su aplicación de marzo a mayo (curso espejo del ciclo escolar 2020-A) y de septiembre a octubre de 2020 (curso espejo del ciclo escolar 2020-B).

El curso de etimologías médicas busca desarrollar en el estudiante, la competencia profesional de entender la terminología médica utilizada en su quehacer profesional, además de desarrollar la habilidad de construir términos médicos aplicados a su campo de estudio. Para apoyar a que el estudiante desarrolle tal competencia se llevó a cabo el curso por medio de Google Classroom, para las actividades que requirieron ser asincrónicas y Zoom en el ciclo escolar 2020-A y Google Meet en el ciclo 2020-B para las sesiones sincrónicas. No se tuvieron alumnos con necesidades educativas especiales por lo que en este sentido no se hicieron adaptaciones. Se realizaron adecuaciones para los alumnos que no contaban con computadora o Internet, a estos, se les enviaron las mismas indicaciones que a sus demás compañeros, solo que por medio de WhatsApp en lugar de Google Classroom, debido a que al menos un integrante de su familia contaba con la aplicación, y las actividades que lo requirieron, las hicieron a mano en alguna hoja y las enviaron por foto por la misma aplicación. Y para subsanar la falta de sesiones sincrónicas con estos alumnos, la autora de la investigación y profesora del curso, envió vídeos con indicaciones y explicaciones que se daban por medio de Zoom y Google Meet a los demás compañeros.

El curso con los cuatro grupos se dividió en cinco etapas: la primera consistió en la elaboración del material didáctico para aprender los prefijos y sufijos por parte de los estudiantes; la segunda, en el aprendizaje de los principales prefijos y sufijos utilizados en las ciencias de la salud; la tercera, en la construcción de términos médicos; la cuarta, en la aplicación de los conocimientos adquiridos; y, finalmente, el cierre del curso por medio de una autoevaluación y retroalimentación por parte de los participantes de la investigación.

Las estrategias de aprendizaje llevadas a cabo de acuerdo a las fases del curso fueron las siguientes:

a) Aprendizaje autónomo: Debido a que el estudiante se encontraba en casa, se le solicitó que revisará un listado de prefijos y sufijos de las ciencias de la salud elaborado por la profesora, autora del presente trabajo. Posteriormente, la o el estudiante debía reflexionar como ha aprendido durante su trayectoria escolar y concientizarse de cómo aprende mejor ¿viendo? ¿escuchando? ¿sintiendo? Para así elaborar su propio material didáctico con los recursos que tenga en casa y de forma libre. El estudiante podía usar toda su creatividad en la elaboración de tal material. Para la realización de esta actividad se podía pedir ayuda de la familia o de alguien con quien conviva.

b) Aprendizaje lúdico: Una vez que se elaboró el material didáctico, se procedió a aprender los principales prefijos y sufijos de ciencias de la salud. Después, se solicitó a los estudiantes que realizaron vídeos en los que algún miembro de la familia, amigo o pareja les preguntaba los principales prefijos y sufijos. También, se pidió la realización de poemas, canciones, chistes, cuentos, refranes en donde se sustituyeron palabras en "español", por prefijos y sufijos en griego y latín, por ejemplo, "cuando mis oftalm te ven, mi cardi late taqui" (cuando mis ojos te ven, mi corazón late rápido). Se dejó como

La familia y el juego como estrategia de aprendizaje a distancia durante la pandemia del Covid19 en México: Una propuesta desde la enseñanza universitaria en ciencias de la salud. Giovanna Georgina Ramírez Cerón. 
tarea, la elaboración de vídeos con la participación de la familia donde los universitarios representaban situaciones de la vida cotidiana sustituyendo palabras en "español" por prefijos y sufijos en griego y latín. Una vez que se logró esta competencia, se pasó al ejercicio de construcción de términos médicos por medio de juegos. Los juegos propuestos por la investigadora fueron: 1) memorama ${ }^{2}, 2$ ) caras y gestos $\left.{ }^{3}, 3\right) ~ ¡$ basta! $^{4}$ de $^{2}$ términos médicos y 4) skeches. Debido a que se dio la libertad al estudiante de diseñar más juegos, algunos de ellos llevaron a cabo monopoly de términos médicos, lotería, ahorcado, uno, jenga, crucigrama, sopa de letras, serpientes y escaleras, por mencionar algunas de las propuestas de los universitarios.

c) Aprendizaje por casos: Una vez que los estudiantes desarrollaron la habilidad de construir y entender terminología médica con el apoyo de su familia, amigos o pareja por medio del juego, se pasó a la etapa en la que aplicaban este aprendizaje al enfrentarse a documentos que encontrarán en su quehacer profesional. Por lo que se le proporcionó a los estudiantes, una serie de ejemplos de casos clínicos y estudios de gabinete y laboratorio para que los leyeran y los "tradujeran" a un lenguaje fácil de entender para la población general. Este ejercicio, lo hicieron para sus familias y posteriormente se solicitó que les dijeran que se siente de verlos entender este tipo de textos.

\footnotetext{
2 Este juego consiste en encontrar pares, los cuales se logran al tener dos fichas idénticas. Se juega por turnos, y en cada uno se voltea una ficha y se memoriza en qué lugar se encontraba, para que, cuando salga su pareja, se pueda formar un par. Gana el participante que tenga más pares al final del juego.
}

${ }^{3}$ A este juego también se le conoce como «adivínelo con señas» 0 "sin palabras». Las instrucciones son: Escribir en papelitos términos médicos que ya se hayan aprendido, posteriormente se doblan para que nadie los vea y se colocan en un recipiente. A través de videollamada con la pareja, amigos o compañeros, o de forma presencial con algún miembro de la familia, se va a sacar uno de los papelitos y SIN HABLAR, quien sacó el papel va a representar el término que le tocó. Por ejemplo, cardiomegalia, con mis manos formo un corazón y extiendo mis brazos para hacerlo grande. La otra persona debe de adivinar qué término es y decir su significado. Si se hace con la familia, se le puede explicar qué significan los términos para hacer el juego más divertido. Si se lleva a cabo a través de alguna plataforma digital, cada jugador saca un papelito desde casa y, sin mostrarlo a los demás, lo representa; los otros jugadores, por medio de la pantalla, intentarán adivinar el término.

${ }^{4}$ Este juego se basa en el ¡basta! clásico, el cual consiste en que uno de los participantes dirá la letra «A» en voz alta, posteriormente, la misma persona, de manera mental, va a decir todo el alfabeto mientras otro de los participantes es el responsable de gritar «ibasta!». Cuando este lo diga, el jugador que estaba pensando el alfabeto informa en qué letra se quedó. Enseguida, los participantes escribirán un nombre, apellido, ciudad, flor o fruto, color, y cosa, iniciando con la letra del alfabeto en la cual se detuvo el jugador que era responsable de pensarlo. La adecuación que se hace en la asignatura de etimologías médicas es que a través de una videollamada o con las personas con que se convive en casa, se va a seguir la misma dinámica, solo que en lugar de decir una letra del alfabeto se van a sacar prefijos y sufijos de una cajita en donde previamente se colocaron en papelitos doblados. En vez de escribir nombre, ciudad, o flor o fruto... se van a construir términos médicos con el prefijo o sufijo que se sacó. Gana el participante que construya más términos médicos sabiendo su significado.

La familia y el juego como estrategia de aprendizaje a distancia durante la pandemia del Covid19 en México: Una propuesta desde la enseñanza universitaria en ciencias de la salud. Giovanna Georgina Ramírez Cerón. Página $9 \mid 20$ 
Autoevaluación: Finalmente, se llevó a cabo el cierre del curso por medio de una autoevaluación, en la que los estudiantes manifestaron como se sintieron, qué fue lo que más les gustó aprender, en criterios cuantitativos cuanto se pondrían de calificación, qué les hizo falta para aprender más y cómo mejorarían los siguientes cursos que se impartan a distancia como consecuencia de la pandemia por Covid-19. Las preguntas realizadas en dicho instrumento, el cual fue elaborado por la autora del presente estudio, se exponen a continuación:

1. ¿Cómo te sientes en cuanto a los conocimientos adquiridos en el curso?

2. ¿Qué fue lo que más te gustó aprender?

3. ¿Cuál es el aprendizaje más significativo que tuviste en el curso? No me refiero solo a lo académico, también puede ser un aprendizaje personal o incluso en tus habilidades para usar la tecnología.

4. ¿Cómo te sentiste al desarrollar las actividades? ¿Cuál fue la actividad que más te gusto y por qué?

5. Comparando las clases en línea con las clases presenciales ¿Qué es lo que más te gusta más de cada una de estas dos modalidades?

6. ¿Cómo consideras que fue tu aprovechamiento en el curso?

7. ¿Qué te hizo falta para aprender más?

8. ¿Qué calificación te pondrías del 0 al 100 ? 0 es la calificación más baja y cien la más alta.

9. ¿Cómo mejorías los siguientes cursos de manera virtual durante la pandemia?

3. Evaluación de la propuesta: Se evaluaron las estrategias de aprendizaje utilizadas en la educación a distancia, durante estos primeros ocho meses desde que se dio el cierre de las escuelas como medida de prevención de contagios por Covid-19. Para evaluar el grado en que los estudiantes adquirieron la competencia a desarrollar en el curso marcada por el programa de estudios, se les solicitó leer un documento de ciencias de salud y explicar el significado de cada término médico encontrado en el texto. Además, se realizó una evaluación cualitativa por medio de un cuestionario en Word enviado a través de Google Classroom, en el que los propios estudiantes participantes evaluaban su desempeño, las estrategias de aprendizaje llevadas a cabo mediante el curso a distancia y manifestaron sus propuestas para mejorar las clases durante las siguientes fases de la pandemia.

4. El análisis de los datos obtenidos por el cuestionario de autoevaluación se analizó mediante una codificación abierta de los datos. Los estudiantes otorgaron su permiso para hacer uso de estas respuestas para la investigación y así mejorar la manera en que se dan los cursos en tiempos de Covid-19.

La familia y el juego como estrategia de aprendizaje a distancia durante la pandemia del Covid19 en México: Una propuesta desde la enseñanza universitaria en ciencias de la salud. Giovanna Georgina Ramírez Cerón. 


\section{Resultados}

De los ciento veinte estudiantes participantes desertaron de sus estudios universitarios once, solo una de estos, informó que le estaba siendo difícil el aprendizaje en medio de la situación sanitaria y se daría de baja de la universidad, los otros diez dejaron de comunicarse y fue imposible contactarlos para conocer el motivo por el cual no concluyeron el curso y dejaron de hacer el envío de sus actividades a partir de la segunda semana del comienzo de la clase. Dos de los alumnos que cursaron la unidad de aprendizaje de etimologías médicas en el primer ciclo escolar de suspensión de clases presenciales, es decir, en el ciclo 2020-A, solicitaron explícitamente a la profesora el "favor" de reprobarlos, porque no tenían Internet en casa, solo datos móviles en su celular. Al conocer la propuesta de la profesora para tomar el curso que consistió en realizar las actividades y enviarlas por WhatsApp, los alumnos con problemas de acceso aceptaron concluir la materia, pues esta alternativa facilitaba la construcción de su aprendizaje y continuidad en la universidad.

El promedio de calificación obtenida por los estudiantes que concluyeron el curso fue de 97.42. La calificación se logró al entregar a la profesora investigadora las evidencias de aprendizaje y demostrar que habían desarrollado la competencia de entender la terminología médica por medio de la interpretación de documentos de ciencias de la salud y la habilidad de explicar su contenido. En la tabla número 2 se recogen los resultados obtenidos con relación a la calificación de los estudiantes.

\section{Tabla 2}

Calificaciones obtenidas por los participantes del estudio

\begin{tabular}{lc}
\hline \multicolumn{1}{c}{ Calificación final } & Número de estudiantes \\
\hline 100 & 82 \\
$96-99$ & 8 \\
$91-95$ & 6 \\
$86-90$ & 8 \\
$80-85$ & 2 \\
$60-79$ & 11 \\
Sin derecho & \\
Total & 120 \\
\hline $\begin{array}{l}\text { Nota: Elaboración propia } \\
\text { * La calificación mínima para aprobar es sesenta. } \\
\text { ** Sin derecho significa que el estudiante no se presentó al } \\
\text { menos en un ochenta por ciento de las sesiones para poder } \\
\text { obtener calificación en ordinario. O en un mínimo de sesenta }\end{array}$
\end{tabular}

La familia y el juego como estrategia de aprendizaje a distancia durante la pandemia del Covid19 en México: Una propuesta desde la enseñanza universitaria en ciencias de la salud. Giovanna Georgina Ramírez Cerón. 
por ciento de las sesiones para tener derecho a la realización de un extraordinario.

En la autoevaluación llevada a cabo por los participantes, estos se asignaron una calificación cuantitativa sobre su desempeño en el curso, de acuerdo con su vivencia. El promedio atribuido fue de 92.77, casi cinco puntos menos que la calificación basada en las competencias demostradas. Los estudiantes que tuvieron un promedio de noventa a cien puntos en su acta de calificación, coincidieron con el puntaje manifestado en su autoevaluación. Los argumentos presentados para justificar su respuesta fueron que entregaron todo, que aprendieron mucho y que le echaron muchas ganas. Por contraste, quienes obtuvieron menos de noventa puntos de calificación en el curso, se asignaron una calificación mucho menor en su autoevaluación, este grupo de estudiantes refirió que merecía esa calificación porque muchas veces no podían acceder a la tecnología y que incluso merecían reprobar. A partir de estos datos se puede observar que el cierre de las escuelas ha dejado al descubierto la desigualdad en el acceso a las tecnologías necesarias para seguir con los procesos educativos durante la pandemia por Covid-19. También se puede inferir, cómo los estudiantes que viven esta brecha digital se responsabilizan a sí mismos por no contar con los recursos necesarios y perciben que merecen una calificación menor a la de sus demás compañeros solo por el hecho de no contar con computadora e Internet.

Treinta y seis de los estudiantes que participaron en la investigación refirieron que se sintieron bien en relación a los conocimientos adquiridos en el curso, veintiséis se sintieron muy satisfechos/as, diecisiete de ellos sintieron que aprendieron, catorce se sintieron contentos/as, trece se sintieron felices, tres no se sintieron satisfechos/as, a dos no les gustó esta modalidad, uno de ellos refirió que fue muy confuso y once no concluyeron el curso. La figura 1 resume estos resultados.

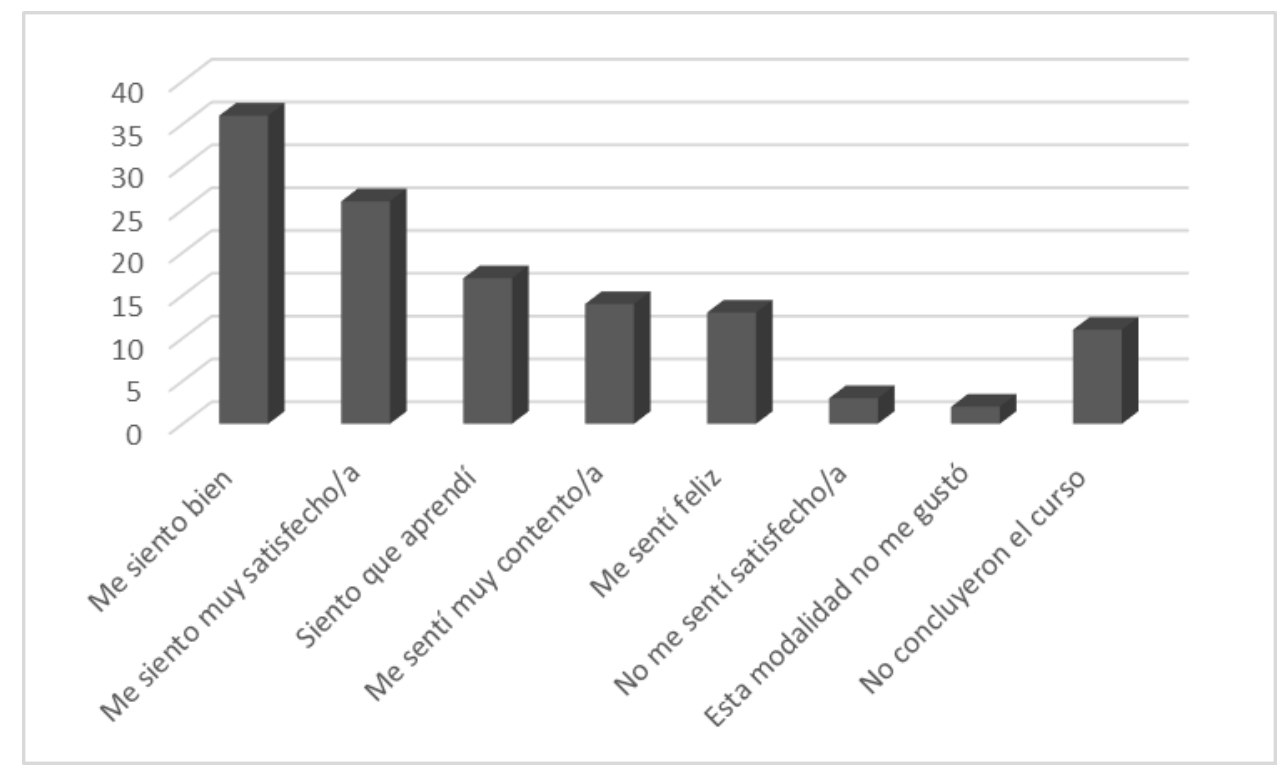

Figura 1. Sensaciones del alumnado participante respecto a los conocimientos adquiridos.

Nota: Elaboración propia

La familia y el juego como estrategia de aprendizaje a distancia durante la pandemia del Covid19 en México: Una propuesta desde la enseñanza universitaria en ciencias de la salud. Giovanna Georgina Ramírez Cerón. 
Lo que más les gustó aprender a las y los estudiantes fue construir términos médicos, entenderlos y aplicarlos, así como poder comprender los ejemplos de casos clínicos de la actividad integradora, ya que desde su percepción, el entendimiento de la terminología utilizada en las ciencias de la salud es esencial para ser incluidos en su práctica profesional y en conversaciones en las que se utilicen. Los participantes eligieron estudiar una profesión de las ciencias de la salud para servir a los demás y a través de la competencia desarrollada en el curso pueden ayudar a otros, especialmente a su familia. También mencionaron que les gustó que se puede aprender de una forma divertida, así como la convivencia con sus compañeros y familiares. Entre las respuestas dadas por los participantes se encuentran:

Lo que más me gustó aprender fue...

"Aprender la formación y significado de los términos"

"Estructurar términos por mí misma"

"El origen de las palabras y como se usan"

"Los términos y que se puede aprender en línea cuando hay compromiso de alumnos y maestros"

"Me gustó mucho aprender a traducir"

"Que entendí el caso"

"Poder entender los casos sin ayuda en internet o ver los apuntes"

"Formar casos clínicos, que no es tan malo trabajar en línea como yo pensaba, lo pude hacer a través de la tecnología"

"Satisfacción de leer los diagnósticos y entenderlos"

"Los términos y ayudar a mi familia"

"Que podía explicarle a mi familia los términos"

"Gratificante que aprendí, en mi familia hay doctores y les podía entender"

"Que mi familia estuvo conmigo en la tarea"

"Gracias a las actividades valoré a las personas que me rodean, me apoyaron en todo lo que llegué a presentar"

"Saber utilizar la tecnología y programas que jamás había utilizado y compartir actividades con mi familia"

"La convivencia en línea con mis compañeras"

La familia y el juego como estrategia de aprendizaje a distancia durante la pandemia del Covid19 en México: Una propuesta desde la enseñanza universitaria en ciencias de la salud. Giovanna Georgina Ramírez Cerón. 
"Formar términos médicos, hacer el vídeo junto con mis compañeras, aprendí que puedo ser una persona muy creativa y puedo adquirir conocimiento poniendo muchas ganas de mi parte"

"Que nos tenemos que acostumbrar a la vida tecnológica, a valorar la vida y el estudio, a valorar el estudio y no rendirme"

"De que a pesar de que no estuve en clases presenciales tuve un buen aprendizaje y con dinámicas divertidas pude aprender"

"Que es muy interesante aprender con dinámicas, es más fácil de aprender y también con los vídeos donde nos explicaba"

"Sacarle provecho a las TIC"S aprender los términos médicos"

"Conocí mejor las tecnologías"

Cabe mencionar que en México, el personal de la salud recibió agresiones por parte de algunos ciudadanos (Organización de las Naciones Unidas, 23 de abril de 2020), entre estas se destacan rocíos con cloro y agresiones verbales bajo el argumento de que ellos propagaban el Covid-19. Seis estudiantes de enfermería del calendario escolar 2020-B refirieron que los actos de violencia contra el personal de la salud les han hecho sentirse tristes y esto ha repercutido en su aprendizaje, puesto que ellas y ellos decidieron estudiar su carrera para servir a la gente y el hecho de ver como "pagan", los ha hecho sentir mal y dudar sobre seguir adelante.

Entre los aprendizajes más significativos se encuentran que "realmente se puede aprender desde casa" (estudiante de enfermería), y que es posible adquirir conocimientos de una manera divertida. También, descubrieron habilidades propias que no conocían, como el manejo de la tecnología en la educación, aprendieron a usar aplicaciones que nunca habían utilizado como Google Classroom, Zoom o Google Meet, se dieron cuenta que tienen la capacidad de aprender desde casa, de que son muy creativos y que tienen una buena capacidad de organización. Sobre todo, en el caso de estudiantes universitarios que son padres y madres de familia, ya que, además de estudiar una carrera a distancia, debían de apoyar a sus hijos con la educación en casa, pues la suspensión de actividades escolares presenciales ha sido en todos los niveles educativos. En algunos casos, les daba vergüenza hacer vídeos, pero con las actividades de la materia se desinhibieron y les agradó continuar con esta actividad en otros contextos, a continuación se cita la respuesta dada por una estudiante de enfermería:

"La actividad que más disfrute fue la de enviar videos con las partes del cuerpo, fue muy divertido, mi sobrina de 9 años me ayudó y cada vez que equivocaba o trababa nos reíamos mucho y comenzamos de nuevo a grabar. Este ejercicio fue además el que me inspiro a comenzar mi canal de YouTube, ya que me demostró que no era tan mala en los videos como creía (en mi quinto video le mande saludos jeje)."

Se encontró que la inclusión de la familia, amigos, compañeros y pareja en las actividades de aprendizaje, tuvo resultados positivos, pues para las y los estudiantes

La familia y el juego como estrategia de aprendizaje a distancia durante la pandemia del Covid19 en México: Una propuesta desde la enseñanza universitaria en ciencias de la salud. Giovanna Georgina Ramírez Cerón. Página 14 | 20 
participantes el hecho de que sus familiares les ayudaran unió más a la familia. En algunos casos la realización de la tarea de etimologías médicas era un momento de diversión como familia donde participaban la mayoría de los integrantes, en otros se trataba de un momento que implicaba tomarlo con toda la seriedad que merece. Una estudiante de enfermería refirió que cuando le leyó el caso clínico a sus seres queridos lo hizo en una cena familiar: "estábamos cenando y me pare en plena cena, les dije que por favor me escucharan y todos se quedaron sorprendidos cuando vieron lo que aprendí". En voz de las y los participantes de la investigación hacer su tarea con sus seres queridos les ayudó a valorarlos en medio de la situación sanitaria que se vive:

"El apoyo de mi familia, mis amigos y mi pareja, fue muy significativo para mí, ya que muchas veces no valoramos que siempre podemos contar con las personas que nos rodean, y no nos damos cuenta de que están ahí" (estudiante de terapia física).

"Gracias a las actividades, valoré a las personas que me rodean, pues ellos me apoyaron en todo lo que llegue a presentarle" (estudiante de enfermería).

"Desarrollé la paciencia y pude convivir más con mi familia" (estudiante de terapia física).

"Valoré que lo más importante que tengo es mi libertad" (estudiante de enfermería).

"Fue muy gratificante ver que mi familia me vio aprender etimologías" (estudiante de enfermería).

Además, las y los participantes del estudio coincidieron en que les gustó poder compartir con sus seres queridos esta experiencia y el hecho de aprender juntos etimologías médicas los unió; pues papás, mamás, hermanos, primos, novios, amigos, cuñados "entendían de que hablaban".

Otro de los aprendizajes significativos para los estudiantes fue que la pandemia y el aprendizaje en casa aumentó su capacidad de resiliencia. Entre las respuestas dadas por los estudiantes se encontraron las siguientes: "si nos lo proponemos, todos somos capaces de hacer y de aprender cosas nuevas casi en cualquier circunstancia", "cuando se quiere, se puede", "a pesar de la experiencia que estamos viviendo, no hay que darnos por vencidos", "todos tenemos la capacidad de lograr aprender a estudiar de diferentes maneras, a pesar de la situación que está pasando", "gracias a la contingencia superé este reto para mí", "superar la adversidad y ponerle todas las ganas al estudio", "aprendí a echarle ganas, porque nada en la vida es seguro, todo puede cambiar en un abrir y cerrar de ojos", "esto me ayudó a usar tecnologías que no conocía, tuvo algo bueno", "como mamá, aprendí a organizarme, eso fue gratificante para mí".

En cuanto a la actividad que más les gustó realizar, las y los participantes señalaron en primer lugar las que implicaron realizar un juego como memorama, lotería, caras y gestos, entre otros, y en segundo lugar fue la comprensión de un texto de ciencias de salud. También mencionaron que lo que más les agradó de hacer en ambas actividades fue el llevarlas a cabo con sus seres queridos, lo cual hizo más llevadero el encierro y el distanciamiento social como medida preventiva a causa de la pandemia por Covid-19. En cuanto a cómo se sintieron los estudiantes participantes al realizar las actividades de

La familia y el juego como estrategia de aprendizaje a distancia durante la pandemia del Covid19 en México: Una propuesta desde la enseñanza universitaria en ciencias de la salud. Giovanna Georgina Ramírez Cerón. 
aprendizaje treinta y seis refirieron haberse relajado, incluso comentaron no sentir que estuvieran realizando una tarea, disfrutaron de las actividades y se sintieron a gusto. Treinta y cuatro, manifestaron haberse sentido bien. Dieciséis sintieron frustración, estrés o preocupación, de los cuales, cuatro manifestaron sentir estrés y frustración por la falta de acceso a la tecnología; los demás no especificaron porque experimentaron estas emociones. Doce se sintieron contentos y emocionados al llevar a cabo las actividades, mientras que seis manifestaron sentir felicidad, una de las estudiantes de enfermería indicó que se sintió "feliz porque nunca hice algo similar". Cinco se sintieron capaces, uno de ellos refirió "me sentía como todo un profesional y me imagine así en unos años", aquí se aprecia la propuesta de Vygotski (1979; 2009), sobre el jugar a ser lo que soy, en la que a través de la interpretación de documentos, algunos estudiantes por medio de su imaginación "jugaban" a ser enfermeros, aunque no lo manifestaron explícitamente con esta terminología. Once no concluyeron el curso. La figura 2 sintetiza los resultados obtenidos.

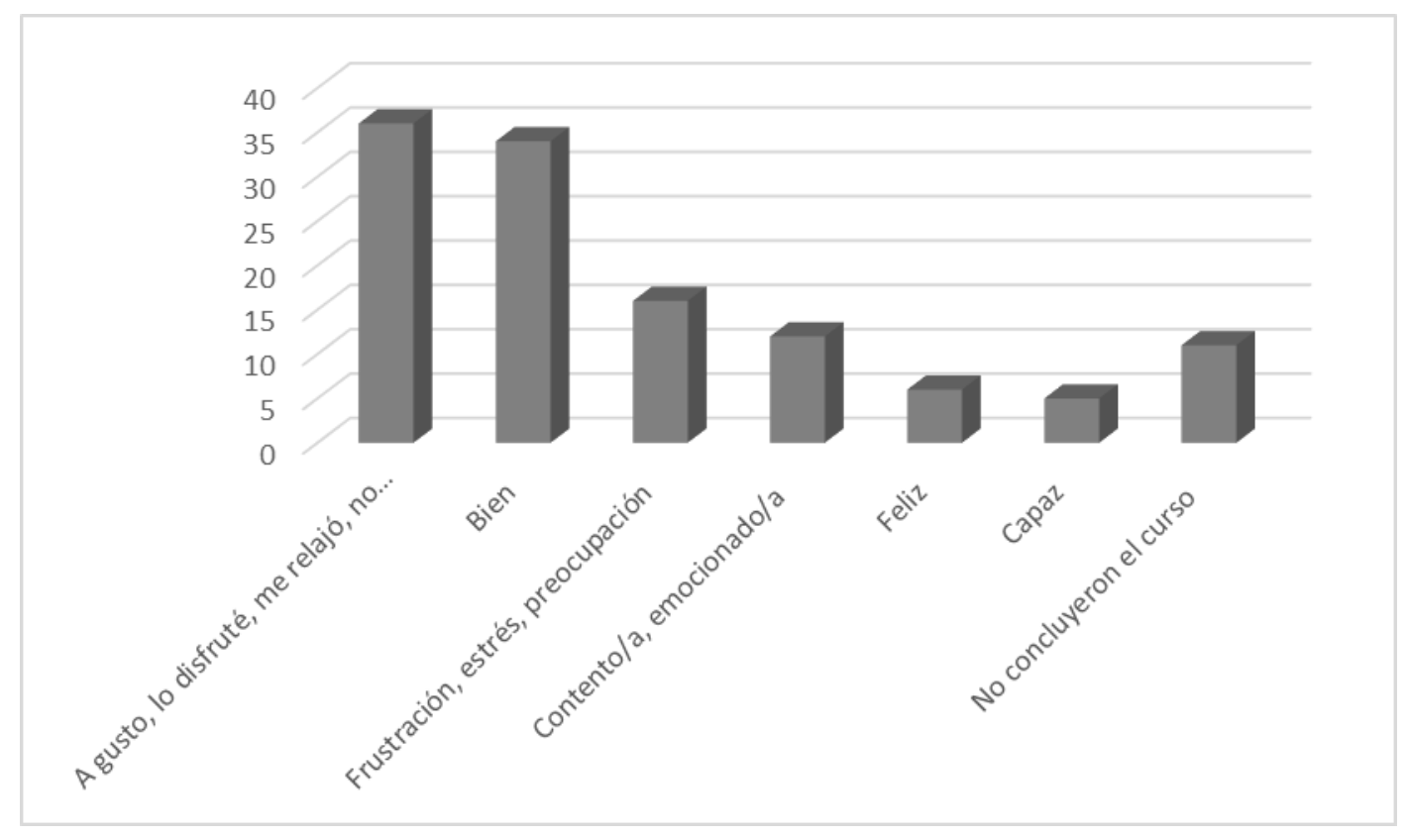

Figura 2. Sensaciones de las y los estudiantes al realizar la actividades. Nota: Elaboración propia.

Finalmente, en la autoevaluación, los estudiantes participantes realizaron propuestas para mejorar las clases a distancia en los siguientes cursos que se impartan a distancia durante la pandemia por Covid-19. Ellas y ellos piden a los profesores realizar más sesiones a través de videoconferencias para que sean resueltas sus dudas y tener más contacto con ellos, y que respeten el horario de las clases presenciales; que ponderen la cantidad de tareas que encargan y que las expliquen adecuadamente, además de que estén pendientes de que hayan quedado claras las indicaciones para su realización; y sobretodo, que sean empáticos y comprensivos con los estudiantes que no tienen fácil acceso a las tecnologías para continuar sus estudios universitarios. Lo que refleja la necesidad de los estudiantes de sentirse cerca de sus profesores, acompañados por estos y comprendidos en la situación que atraviesan.

La familia y el juego como estrategia de aprendizaje a distancia durante la pandemia del Covid19 en México: Una propuesta desde la enseñanza universitaria en ciencias de la salud. Giovanna Georgina Ramírez Cerón. 


\section{Conclusiones}

A partir de los resultados expuestos, se puede concluir que la competencia a desarrollar establecida en el programa de estudio de la asignatura de etimologías médicas, fue alcanzada a través de las estrategias de aprendizaje llevadas a cabo en el presente estudio en la modalidad a distancia durante los primeros ocho meses de la pandemia por Covid-19 en México, las cuales incluyen el juego y la participación de la familia como parte de la Zona de Desarrollo Próximo de los participantes.

Aunque las tecnologías de la información y la comunicación han constituido el espacio de encuentro entre profesores y alumnos para dar continuidad al aprendizaje a distancia, es de suma importancia considerar el contexto en el que están aprendiendo las y los estudiantes participantes. Es decir, un ambiente de incertidumbre sobre lo que va a pasar tanto escolar, como económica y sanitariamente, incertidumbre frente a un regreso a las aulas que parece que nunca va a suceder. Todo esto en medio del confinamiento, del distanciamiento social y de las consecuencias psicosociales que trae consigo como estrés, ansiedad y depresión, así como la falta de acceso a la tecnología. Además de lo anterior, los participantes de la investigación son estudiantes de ciencias de la salud, y aprenden desde casa viendo cómo en su Estado y en su país los profesionales de la salud viven agresiones ya que son vistos por algunos ciudadanos como portadores del virus, lo cual ha mermado en algunos estudiantes la motivación por aprender. El hecho de que para los estudiantes fue tan importante comprender los términos médicos como aprender a salir adelante en medio de esta pandemia, da cuenta de la importancia que tiene este contexto de aprendizaje para los estudiantes. En este sentido, es destacable que la mayoría de los estudiantes mostraron una alta capacidad de resiliencia frente a la situación.

Diseñar una estrategia de aprendizaje en la que se considere este contexto incluyendo a la familia y el juego para contribuir a que la pandemia sea más llevadera para las y los estudiantes, tuvo un efecto altamente positivo en el desarrollo de los aprendizajes necesarios para dar continuidad a la educación universitaria. Por ello, se propone implementar actividades lúdicas y la participación de la familia y seres queridos con quienes convivan los universitarios en casa para mitigar los efectos psicosociales como consecuencia de las medidas de prevención de contagios y así mejorar los procesos de aprendizaje.

La asignatura de etimologías médicas es teórico-práctica, y los resultados fueron positivos en una materia de esta naturaleza, sin embargo, es importante estudiar cómo continuar aprendiendo en casa con cursos de naturaleza totalmente práctica, como por ejemplo, la materia de disección en estudiantes de medicina.

Por otra parte, es importante no olvidar la brecha digital que se sigue experimentado, y ser empáticos con los estudiantes que no puedan tener acceso a la tecnología; casi el diez por ciento de los estudiantes participantes desertaron de la universidad, aún hace falta más trabajo para que la educación verdaderamente sea un derecho.

Se sugiere al profesorado apertura a las nuevas formas de enseñanza-aprendizaje que se han tenido que implementar para que las y los estudiantes no interrumpan su formación

La familia y el juego como estrategia de aprendizaje a distancia durante la pandemia del Covid19 en México: Una propuesta desde la enseñanza universitaria en ciencias de la salud. Giovanna Georgina Ramírez Cerón. 
durante la pandemia por Covid-19, ya que no es posible limitarse a impartir una clase como se hacía antes de la pandemia, sólo que ahora detrás de una pantalla. Es importante emplear toda la creatividad para que las y los estudiantes puedan aprender allá en casa, en medio del encierro y las repercusiones por el Covid-19, por lo que se requiere de empatía y flexibilidad, sobre todo con quienes no tienen los recursos económicos y materiales para aprender a distancia.

Finalmente, se propone que en las etapas venideras de la pandemia se busque mayor cercanía con las y los estudiantes a pesar de la distancia, esto se puede lograr a través de videollamas o videoconferencias, ellas y ellos necesitan sentir que no aprenden solos, y que detrás de la pantalla se encuentran los docentes para apoyarlos en su formación académica. Se sugiere realizar investigaciones sobre el impacto que ha tenido la modalidad a distancia para las y los profesores, además de trabajos que brinden herramientas para que estos puedan mejorar su práctica durante la pandemia, pues a nivel mundial son quienes hacen frente al Covid-19 desde las aulas, aunque en estos momentos deban ser virtuales por el bien de todas y todos.

Presentación del artículo: 14 de noviembre de 2020

Fecha de aprobación: 28 de diciembre de 2020

Fecha de publicación: 08 de enero de 2021

Ramírez Cerón, G.G. (2021). Estrategias de aprendizaje a distancia durante la pandemia del Covid-19 en México: Una propuesta desde la enseñanza universitaria en ciencias de la salud. Revista Educación a Distancia (RED), 21(65). https://doi.org/10.6018/red.456231

\section{Financiación}

Este trabajo no ha recibido ninguna subvención específica de los organismos de financiación en los sectores públicos, comerciales o sin fines de lucro.

\section{Referencias}

Blanco, S. (26 de abril de 2020). Suman 74 personas arrestadas por no usar cubrebocas. El Informador. Recuperado de https://www.informador.mx/jalisco/Suman-74personas-arrestadas-por-no-usar-cubrebocas-20200426-0010.html

Bernabeu, N. y Goldstein, A. (2009). Creatividad y aprendizaje. El juego como herramienta pedagógica. Narcea.

Centro Universitario de Ciencias de la Salud. (2020). Misión. Recuperado de http://www.cucs.udg.mx/nuestro-centro/mision

La familia y el juego como estrategia de aprendizaje a distancia durante la pandemia del Covid19 en México: Una propuesta desde la enseñanza universitaria en ciencias de la salud. Giovanna Georgina Ramírez Cerón. 
Comisión Económica para América Latina y el Caribe-Organización de las Naciones Unidas para la Educación, la Ciencia y la Cultura CEPAL-UNESCO. (2020). La educación en tiempos de la pandemia de COVID-19. Editorial CEPAL, UNESCO.

Crovi, D. (2006). Educar en la era de las redes. Ciudad de México: UNAM.

Elliott, J. (2005). La investigación-acción en educación. Madrid: Ediciones Morata.

Gobierno del Estado de Jalisco. (15 de marzo de 2020). A diferencia de la decisión que tomó el Gobierno de México, en Jalisco las clases en educación básica se suspenden a partir del próximo martes 17 de marzo. Recuperado de https://www.jalisco.gob.mx/es/prensa/noticias/102604

Mendiguren, T., Meso, K., y Pérez, J. A. (2012). El uso de las redes sociales como guía de auto aprendizaje en la Facultad de comunicación de la UPV/EHU. Monográfico, $6,107-122$.

Montes de Oca, R. (2007). Alfabetización múltiple en nuevos ambientes de aprendizaje. Universidad Juárez Autónoma de Tabasco.

Olmedo, N. y Farrenons, V. (2017). Modelos constructivistas de aprendizaje en programas de formación. Catalunya: OmniaScience.

Organización de las Naciones Unidas. (23 de abril de 2020). La ONU en México condena las agresiones contra los profesionales de la salud que luchan contra el coronavirus. Recuperado de https://news.un.org/es/story/2020/04/1473372

Ortíz, A. (2009). Lúdica y creatividad familiar para el crecimiento y desarrollo humano de tus hijos. Ediciones CEPEDID.

Periódico Oficial del Estado de Jalisco (17 de marzo de 2020). Acuerdo del ciudadano gobernador constitucional del estado libre y soberano de Jalisco mediante el cual se clausuran de manera temporal salones de fiesta, casinos, antros, cantinas, centros nocturnos y bares, derivado de la pandemia de Covid-19. Recuperado de https://periodicooficial.jalisco.gob.mx/sites/periodicooficial.jalisco.gob.mx/files/0317-20-vi.pdf

Piscitelli, A. y Adaime, I. (2010). Introducción. En A. Piscitelli, I. Adaime, e I. Binder (Comps.), El proyecto Facebook y la posuniversidad. Sistemas operativos sociales y entornos abiertos de aprendizaje (pp. XV-XX). Madrid, España: Fundación Telefónica.

Ponce, M. E. (2016). La autogestión para el aprendizaje en estudiantes de ambientes mediados por la tecnología. Diálogos sobre educación, 12, 1-23. https://doi.org/10.32870/dse.v0i12.258.

Ramírez-Ortiz, J., Castro-Quintero, D., Lerma-Córdoba, C., Yela-Ceballos, F., y Escobar-Córdoba, F. (2020). Consecuencias de la pandemia Covid-19 en la salud

La familia y el juego como estrategia de aprendizaje a distancia durante la pandemia del Covid19 en México: Una propuesta desde la enseñanza universitaria en ciencias de la salud. Giovanna Georgina Ramírez Cerón. Página $19 \mid 20$ 
mental asociadas al aislamiento social. Recuperado de https://preprints.scielo.org/index.php/scielo/preprint/view/303/358.

Secretaría de Gobernación. (2020a). Acuerdo por el que se establecen las medidas preventivas que se deberán implementar para la mitigación y control de los riesgos para la salud que implica la enfermedad por el virus SARS-CoV2 (COVID-19). Recuperado https://www.dof.gob.mx/nota_detalle.php?codigo=5590339yfecha=24/03/2020

Secretaría de Gobernación. (2020b). Acuerdo por el que se modifica el similar por el que se establecen acciones extraordinarias para atender la emergencia sanitaria generada por el virus SARS-CoV2, publicado el 31 de marzo de 2020. Recuperado de https://www.dof.gob.mx/nota_detalle.php?codigo=5592067yfecha=21/04/2020

Secretaría de Salud. (28 de febrero de 2020). Se confirma en México caso importado de coronavirus COVID-19. Recuperado de https://www.gob.mx/salud/prensa/077-seconfirma-en-mexico-caso-importado-de-coronavirus-covid-19

Secretaría de Salud. (2020). Semáforo Covid-19. Recuperado de https://coronavirus.gob.mx/semaforo/

Universidad de Guadalajara (16 de marzo de 2020). Circular número 4. Recuperado de http://www.udg.mx/sites/default/files/adjuntos/circular_04_2020.pdf

Vygotski, Lev S. (1979; 2009). El desarrollo de los procesos psicológicos superiores. Barcelona: Crítica.

La familia y el juego como estrategia de aprendizaje a distancia durante la pandemia del Covid19 en México: Una propuesta desde la enseñanza universitaria en ciencias de la salud. Giovanna Georgina Ramírez Cerón. 